\title{
REFINED DETERMINATION OF BREAKPOINTS OF THE TRANSLOCATION $t(1 ; 7)$ ASSOCIATED WITH SIGNS OF HMC SYNDROME
}

\author{
Tatsuro IKeuchi, ${ }^{1}$ Nobuyoshi Motohashi, ${ }^{2}$ Kohtaro Yamamoto, ${ }^{3}$ \\ and Takayuki KuroDA ${ }^{2}$ \\ ${ }^{1}$ Department of Cytogenetics, and ${ }^{3}$ Department of Virology and Immunology, \\ Medical Research Institute, and ${ }^{2}$ Department of Orthodontics II, School of Dentistry, \\ Tokyo Medical and Dental University, Bunkyo-ku, Tokyo 113, Japan
}

\begin{abstract}
Summary High-resolution band analysis was performed in order to precisely determine the breakpoints of a de novo chromosome translocation, $\mathbf{t}(1 ; 7)$, which is associated with clinical signs of HMC syndrome (McKusick's \#239800). The breakpoints were found to be at 1q31.2 and 7p15.1-p15.3, respectively. The finding of the translocation in this case might not be coincidental, but rather suggestive of the gene locus responsible for the development of HMC syndrome at either site of the breakpoints.
\end{abstract}

Key Words HMC syndrome, de novo translocation, 1q/7p, highresolution banding

\section{INTRODUCTION}

The hypertelorism, microtia, facial clefting (HMC) syndrome (registered as \#239800 in McKusick's Mendelian Inheritance in Man, 1990) was first described in two sibs by Bixler et al. (1969), but there have since been only a few reported patients with this syndrome (Schweckendiek et al., 1976; Baraitser, 1982). Recently, we described a Japanese boy diagnosed as having probable HMC syndrome who had a constitutional and apparently balanced de novo chromosome translocation, $t(1 ; 7)$ (Motohashi et al., 1985). Since a causal relationship might exist between the translocation and the phenotypic abnormalities in this case, as recently proposed in many other cases or families (Harper et al., 1989), precise determination of the translocation breakpoints seems necessary in order to assign the gene responsible for this genetic disorder.

Received March 19, 1991; Accepted April 1, 1991. 


\section{CASE REPORT}

The patient, a Japanese boy aged 7.5 years when examined previously, was the second child of healthy and nonconsanguineous parents. He was born at term after an uneventful pregnancy with a birth weight of $2,900 \mathrm{~g}$. At the time of his birth, the mother was 30 and the father was 31 years old. Details of the clinical manifestations of the patient have been described elsewhere (Motohashi et al., 1985). Briefly, he showed the following clinical symptoms: cleft palate, micrognathia, platybasia, short anterior face, ear anomalies such as microtia, extra ear tags and atresia of the auditory canal, eye anomalies including orbital hypertelorism, hypoplastic eye lid, distichia, and alacrimal duct. His psychomotor development was almost within normal limits, although the onset of speech was reported to be delayed. His parents were found to have a normal karyotype.

\section{RESULTS AND DISCUSSION}

In this study, high-resolution banding analysis at the $550-850$ band stage was performed by application of the ethidium bromide method (Ikeuchi, 1984) to PHAstimulated lymphocyte cultures from the patient. Several high-resolution banded chromosomes were analyzed, and the representative partial karyotype is shown in Fig. 1. In the der(1) chromosome, the $1 \mathrm{q} 31.1$ sub-band was retained. The $1 \mathrm{q} 31.3$ sub-band and its distal regions of the normal chromosome 1 were translocated onto the der(7) chromosome. This means that the breakpoint on $1 \mathrm{q}$ was at 1q31.2. In the $\operatorname{der}(7)$ chromosome, band $7 \mathrm{p} 14$ was normally divided into the two sub-bands, $7 \mathrm{p} 14.1$ and $7 \mathrm{p} 14.3$, and the region distal to band $7 \mathrm{p} 21.1$ was unequivocally translocated to $1 \mathrm{q}$. However, the subtle sub-band $7 \mathrm{p} 15.2$ could not be identified on either the $\operatorname{der}(1)$ or $\operatorname{der}(7)$ chromosomes. Presumably, the breakpoint on $7 \mathrm{p}$ would have been at $7 \mathrm{p} 15.2$ or its flanking site. Thus, the patient's karyotype was designated $46, \mathrm{XY}, \mathrm{t}(1 ; 7)(\mathrm{q} 31.2 ; \mathrm{p} 15.1-\mathrm{p} 15.3)$, although another possibility of minute deletion of chromosome material at $7 \mathrm{p} 15.2$ cannot be entirely excluded.

To date, only one report of a chromosome study on HMC syndrome has been made, in which the normal G-banded karyotype was shown in two monozygotic twin patients (Schweckendiek et al., 1976). As far as we know, there have been no reported cases which showed chromosome rearrangements involving either the $1 \mathrm{q} 31$ or $7 \mathrm{p} 15$ segments in association with clinical features comparable to those of our patient.

The association of the clinical signs with a chromosome rearrangement in the present case might not be coincidental, but rather suggestive of the location of gene(s) responsible for the development of HMC syndrome. Thus, the gene locus might be situated at one of the two translocation breakpoints. However, the fact 


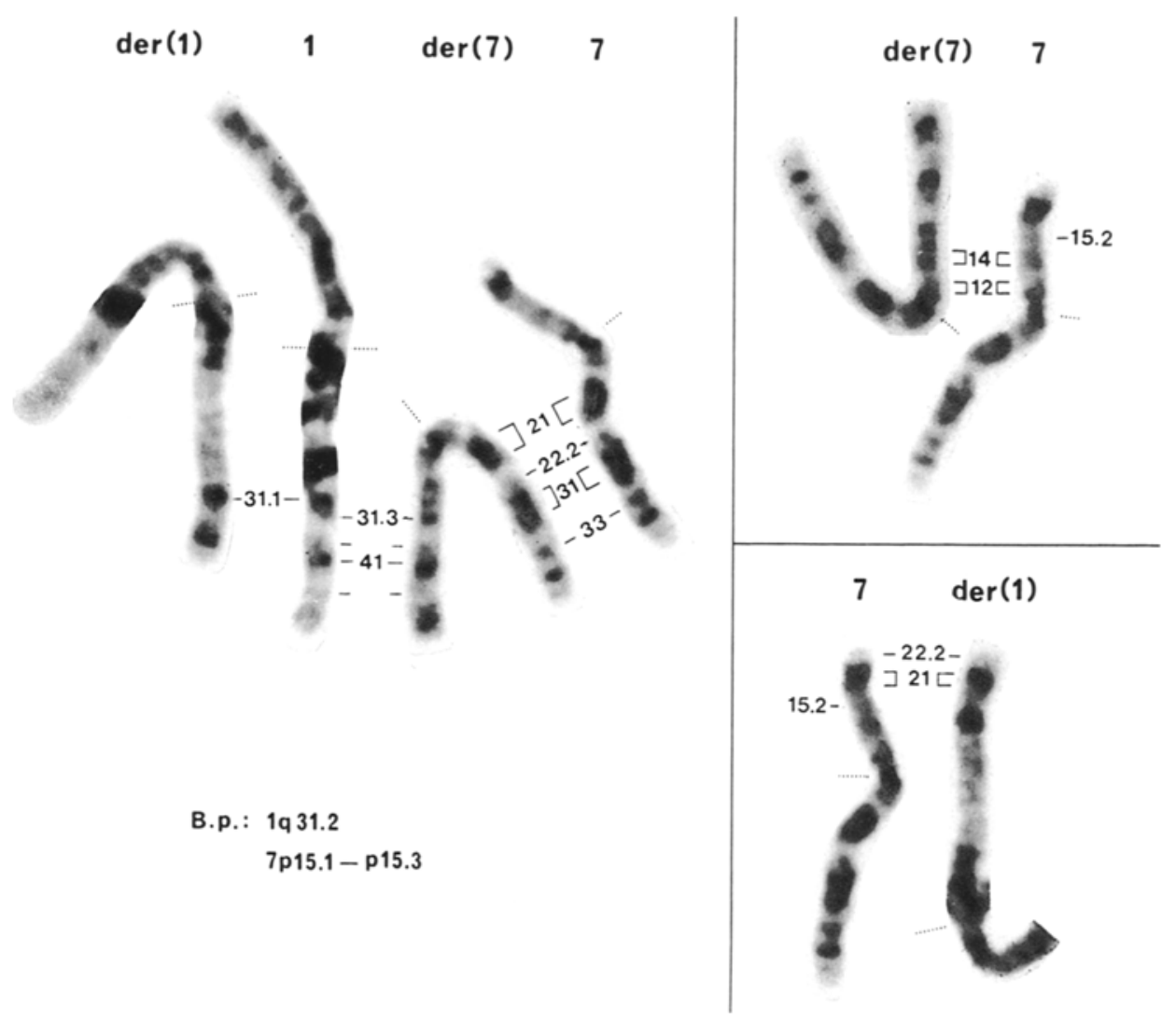

Fig. 1. High-resolution G-banded chromosomes 1 and 7 from the patient. Dotted short lines indicate the centromeres. In order to show the banding homology between the normal no. 7 and the $\operatorname{der}(7)$ or $\operatorname{der}(1)$ chromosomes, two of each chromosome are arranged separately on the right. B.p., breakpoints.

that the patient was a translocation heterozygote is not in agreement with the proposed mode of recessive inheritance in HMC syndrome (Bixler et al., 1969; McKusick, 1990). It remains unknown whether there is any genetic heterogeneity in this syndrome or whether in our case another mutation might exist at the same locus of the normal homologous chromosomes. Further chromosome studies on comparable patients are certainly needed to clarify the effect of the present chromosome translocation.

We have established a lymphoblastoid cell line by EB virus-mediated transformation of the patient's peripheral lymphocytes. This should contribute to further delineation or characterization of the translocation breakpoints at the molecular level, and also to regional gene mapping in general on chromosomes 1 and 7 .

Note added in proof: After this paper had been submitted, we found an article 
(Murray et al., 1990, Am. J. Hum. Genet. 46: 486-491) demonstrating that an autosomal dominant form of cleft palate is tightly linked with the renin locus that maps to chromosome $1 \mathrm{q}$ at the site close to one of the translocation breakpoints in our patient.

Acknowledgments This work was supported in part by a Grant-in-Aid for Scientific Research from the Ministry of Education, Science and Culture, and by Special Coordination Funds for Promotion of Science and Technology from the Science and Technology Agency, Japan.

\section{REFERENCES}

Baraitser, M. 1982. The hypertelorism microtia clefting syndrome. J. Med. Genet, 19: 387-388. Bixler, D., Christian, J.C. and Gorlin, R.J. 1969. Hypertelorism, microtia, and facial clefting: a newly described inherited syndrome. Am. J. Dis. Child. 118: 495-498.

Harper, P.S., Frezal, J., Ferguson-Smith, M.A. and Schinzel, A. 1989. Report of the committee on clinical disorders and chromosomal deletion syndrome. Cytogenet. Cell Genet. 51: 563611.

Ikeuchi, T. 1984. Inhibitory effect of ethidium bromide on mitotic chromosome condensation, and its application to high-resolution chromosome banding. Cytogenet. Cell Genet. 38: 56-61.

McKusick, V.A. 1990. Mendelian Inheritance in Man. Catalogs of Autosomal Dominant, Autosomal Recessive, and X-Linked Phenotypes, 9th Ed., The Johns Hopkins Univ. Press, Baltimore and London.

Motohashi, N., Kuroda, T. and Ikeuchi, T. 1985. A case report: hypertelorism, microtia, cleft palate with a de novo balanced chromosome translocation, $\mathrm{t}(1 \mathrm{q}-; 7 \mathrm{p}+)$. Cong. Anomal. 25: $181-190$.

Murray, J.C., Nishimura, D.Y., Buetow, K.H., Ardinger, H.H., Spence, M.A., Sparkes, R.S., Falk, R.E., Falk, P.M., Gardner, R.J.M., Harkness, E.M., Glinski, L.P., Pauli, R.M., Nakamura, Y., Green, P.P. and Schinzel, A. 1990. Linkage of an autosomal dominant clefting syndrome (van der Woude) to loci on chrosome 1q. Am. J. Hum. Genet. 46: 486-491.

Schweckendiek, W., Hillig, U., Kruse, E., Redeck, G. and Wendt, C.G. 1976. HMC syndrome in identical twins. Hum. Genet. 33: 315-318. 